\title{
Diagnosis and Treatment of Tuberous Sclerosis Manifestations in Children: A Multicenter Study
}

\author{
Marina Flotats-Bastardas ${ }^{1,2}$ Daniel Ebrahimi-Fakhari ${ }^{1}$ Ludwig Gortner ${ }^{3,4}$ Martin Poryo ${ }^{5}$ \\ Michael Zemlin ${ }^{3}$ Alfons Macaya-Ruiz ${ }^{2,6}$ Sascha Meyer ${ }^{1}$
}

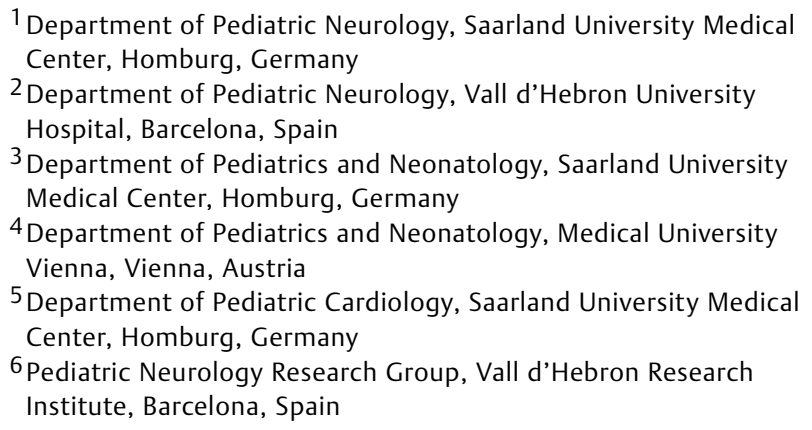

Address for correspondence Marina Flotats-Bastardas, MD, Department of Pediatric Neurology, Saarland University Medical Center, Building 9, Kirrberger Strasse, 66421 Homburg, Saarland, Germany (e-mail: marina.flotats-bastardas@uks.eu).

Neuropediatrics 2018;49:193-199.

\begin{abstract}
Tuberous sclerosis complex (TSC) is a genetic disease with a significant morbidity and mortality. We conducted a retrospective analysis of two cohorts (Vall d'Hebron University Hospital [HVH], Barcelona, Spain, 1982-2015, and at Saarland University Medical Center [UKS], Homburg, Germany, 1998-2015) to assess prevalence and treatment of TSC associated manifestations and to evaluate if the follow-up was in line with published recommendations. This was considered if more than $15 \%$ of patients did not receive adequate examination with regard to potential organ involvement. A definite diagnosis was made in 52 patients (96\%), and a possible diagnosis was made in 2 patients (4\%). Thirty-four (63\%) patients were from $\mathrm{HVH}$ and 20 (37\%) from UKS. Median age at first presentation was 6 months (interquartile range: $0-38$ months), and median time of follow-up was 6 years (interquartile range: 2-13 years). Clinical symptoms that led to a diagnosis of TSC were cardiac

Keywords

- mTOR

- everolimus

- SEGA

- angiomyolipoma

- rhabdomyoma

- epilepsy rhabdomyoma (22/54), epilepsy (20/54), and cutaneous manifestations (4/54). Assessment of neuropsychiatric, renal, and ocular manifestations was inadequate in both hospitals, whereas cutaneous manifestation was inadequate at UKS only. Our data demonstrate insufficient examinations in a substantial number of TSC patients with regard to neuropsychiatric, renal, ocular, and cutaneous manifestations. The recently published guidelines may prove valuable in establishing a more comprehensive approach.
\end{abstract}

\section{Introduction}

Tuberous sclerosis complex (TSC) is a genetic disease often with in utero onset, which is characterized by benign tumors affecting multiple organs including the heart, central nervous system, skin, kidney, and lungs. ${ }^{1-3}$ The broad range of clinical manifestations was first described in the late 19th century and has consequently led to the publication of several consensus received

October 24, 2017

accepted after revision

February 6, 2018

published online

March 20, 2018
(C) 2018 Georg Thieme Verlag KG Stuttgart · New York
DOI https://doi.org/

10.1055/s-0038-1637738. ISSN 0174-304X. 
guidelines with the aim of streamlining the diagnostic and therapeutic approach of TSC-related organ manifestations. ${ }^{4-8}$ The discovery of mutations in the TSC1 and TSC2 genes ${ }^{9,10}$ and their association with the mechanistic target of rapamycin (mTOR) pathway was the basis for the development of a targeted therapy in TSC patients. ${ }^{11} \mathrm{~A}$ large number of randomized controlled clinical trials with MTOR inhibitors have shown efficacy in the treatment of several clinical organ manifestations of TSC, most importantly in patients with subependymal giant cell astrocytoma (SEGA), seizures (treatment-resistant with focal-onset), and angiomyolipoma (AML), as well as cutaneous manifestations. ${ }^{12-16}$

The main objectives of this study were to characterize two samples of TSC patients who attended two different university hospitals (Barcelona, Spain, and Homburg, Germany) with regard to the clinical manifestations of TSC and to assess the quality of the diagnostic and therapeutic approach with regard to published recommendations for diagnosis and surveillance of TSC patients.

\section{Patients and Methods}

This is a retrospective, multicenter study of TSC patients seen at the Pediatric Neurology Service of Vall d'Hebron University Hospital (HVH), Barcelona, Spain (January 1982 to May 2015), and the Department of Pediatric Neurology at Saarland University Medical Center (UKS), Homburg, Germany (May 1998 to May 2015).

Patients with a possible or definitive diagnosis of TSC based on available current diagnostic criteria at the time were included. ${ }^{4,5,7}$ Patients with insufficient data documentation were excluded. All patient files, both handwritten and electronic charts, were reviewed to assess all TSC relevant clinical manifestations, the initial diagnostic approach, and further surveillance management, and whether they were done in line with available current clinical guidelines and recommendations at the time. ${ }^{6,8}$ These included cardiological examination (electrocardiogram and echocardiography), neurologic assessment including brain imaging (cranial computed tomography [CT] or cranial magnetic resonance imaging [MRI]) and electroencephalography (EEG) studies, neurodevelopmental as well as neuropsychiatric evaluation, renal assessment including kidney ultrasound or MRI, and ophthalmological and dermatological examination. ${ }^{6}$ Adequate cardiological examination was considered if at least one documented echocardiogram or electrocardiogram was performed. Brain imaging was reviewed, and TSC-related manifestations (e.g., SEGA, subependymal nodule [SEN], cerebellar tubers, radial migration lines, and cortical cysts) were recorded at initial presentation as well as over time. Adequate brain imaging was defined as at least one performed cranial MRI or CT. SEGA was defined as an SEN more than $1 \mathrm{~cm}$ in size or with serial growth over time, or causing obstructive hydrocephalus. ${ }^{17}$ The occurrence of epilepsy, in particular infantile spasms and treatment with antiepileptic drugs (AEDs), was assessed. Refractory epilepsy was defined if seizures persisted despite treat- ment with $\geq 3$ AEDs. Adequate seizure control was defined as a seizure-free period of at least 12 months upon last follow-up. ${ }^{18}$ A patient was considered to have developmental delay in case of an abnormal age-appropriate neurodevelopmental test or an intelligence quotient test of $\leq 85$, or if the patient experienced a delay in two out of four domains of normal development. Neuropsychiatric manifestation was determined based on neuropsychiatric testing or whenever a diagnosis of autism spectrum disorder or attention deficit/hyperactive disorder syndrome was documented. Ophthalmological and dermatological examinations were considered adequate if results from these examinations were documented in written form in the medical charts.

Results from genetic testing as well as therapy with mTOR inhibitor were included in this study as available. For both cohorts, we assumed that a specific organ manifestation was inappropriately assessed if more than $15 \%$ of the patients did not receive adequate clinical management with regard to specific organ manifestation (diagnosis and follow-up). Furthermore, we compared these data between two study periods (prior to and after 2006).

For patients treated with mTOR inhibitors, both treatment duration and response were assessed. If available, results from genetic testing for mutations in the TSC1 or TSC2 gene was also included into our data analysis.

All data were analyzed with IBM-SPSS Version 22 (SPSS Inc., Chicago, Illinois, United States) using nonparametric tests (qualitative variables with chi-square test, and quantitative variables with Mann-Whitney U test). Data are presented as mean, median, standard deviation, interquartile range, and range as appropriate. Statistical significance was assigned if $p$-value was $<0.05$.

\section{Results}

\section{General Demographics}

Initially, 58 patients were identified, 4 of whom were excluded because of inappropriate medical records or not meeting diagnostic criteria for TSC. A definite diagnosis was made in 52 (96\%) patients and a possible diagnosis was made in $2(4 \%)$ patients (29 [54\%] males and 25 [46\%] females; 34 [63\%] patients in $\mathrm{HVH}$ and 20 [37\%] patients in UKS). The median age at first presentation was 6 months (interquartile range: $0-38$ months), and the median time of follow-up was 6 years (interquartile range: $2-13$ years) (- Supplementary Fig. S1). There was no statistical difference in terms of patient distributions and prevalence of symptoms between the two hospitals.

Results of molecular analysis were available in 31/54 (57\%) patients: $23 / 31$ (74\%) patients demonstrated mutations in the TSC2 gene, 5/31 (16\%) patients demonstrated mutations in TSC1 gene, and 3/31 (10\%) patients were without TSC1/TSC2 gene mutation.

mTOR inhibitors were given to $12 / 54$ (22\%) patients (11 everolimus, 1 sirolimus) at an average age of 10 years (standard deviation: 6.9; range 0-24). Patients were treated on average for 28 months (range: 6-50 months). 


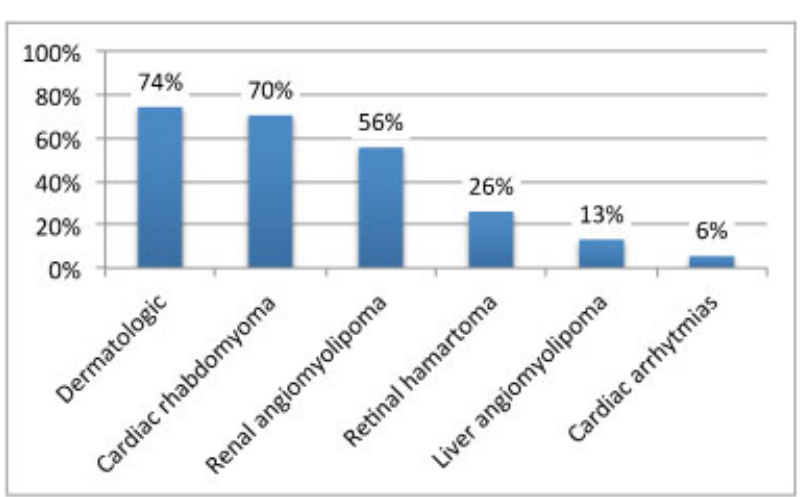

Fig. 1 Summary of the main clinical symptoms in our cohort (other central nervous system).

\section{TSC Nonneurologic Manifestations, Treatment, and Response}

-Figs. 1 and 2 summarize the main clinical symptoms.

The most frequent manifestation that led to a diagnosis of TSC was the presence of cardiac rhabdomyoma (22/54 [41\%] patients, 16 of whom were diagnosed by prenatal ultrasound). Overall, cardiac rhabdomyomas were diagnosed in $38 / 54$ (70\%) patients. In 9/38 (24\%) patients, involution of rhabdomyomas was noted during follow-up visits. Cardiac arrhythmias were seen in 3/54 (6\%) patients.

The existence of renal AML was diagnosed in 30/54 (56\%) patients with a median age of 9 years (interquartile range 610 years), and in 3 of them, the following treatment was given: nephrectomy in 1 , embolization in 1 , and mTOR inhibitor (sirolimus) in 1 . The patient who received sirolimus experienced a reduction of renal AML size.

Liver AML were seen in seven patients. Retinal hamartomas were seen in 14 patients at a median age of 3 years (interquartile range: $0-8$ years).

The cutaneous manifestations were as follows: white spots (35 patients), facial angiofibroma (26 patients), shagreen patch (12 patients), fibrous cephalic plaque (9 patients), gingival fibroma (6 patients), ungual fibroma (4 patients), and dental pits (1 patient). A statistically significant association was found between TSC2 mutations and the occurrence of facial angiofibromas $(p=0.001)$, fibrous cephalic plaque $(p=0.008)$, and shagreen patch $(p=0.014)$.

\section{Epilepsy Manifestations and Treatment}

Epilepsy was the second most common manifestation that led to a diagnosis of TSC (20/54 [37\%] patients). During the follow-up period, a total of 38/54 (70\%) patients had epilepsy. The average age at first onset of seizures was less than one 1 year (interquartile range: $0-1$ years). Infantile spasms were seen in 14/38 (37\%) patients and focal seizures in 24/38 (63\%) patients. Patients were treated with $\geq 3$ AEDs on average (median: 3; range: 0-12). Refractory epilepsy was diagnosed in 20/38 (53\%) patients. - Fig. 3 depicts the use of AEDs depending on hospital location. - Fig. 4 shows which AED medications were given to those patients who achieved good seizure control (15/38 [40\%] patients); 13 of them were under monotherapy. Of the 14 patients with infantile

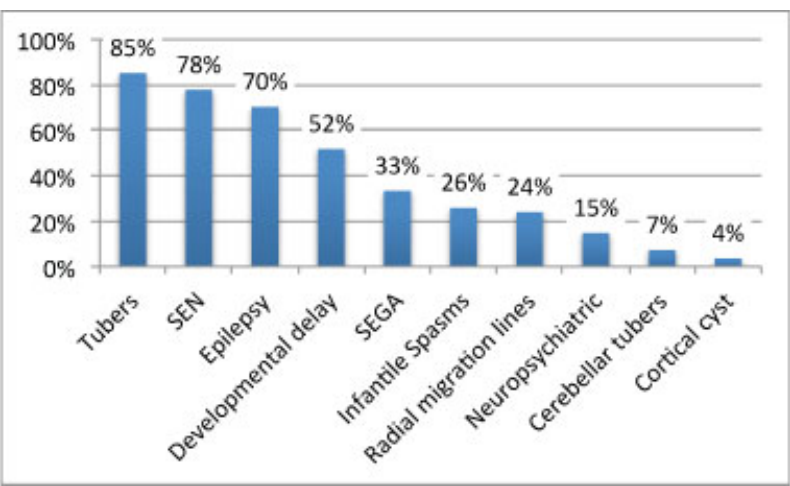

Fig. 2 Summary of central nervous system clinical-radiological manifestations. SEGA, subependymal giant cell astrocytoma; SEN, subependymal nodule.

spasms, 6 (43\%) patients achieved good seizure control. Sulthiame was the only AED with a statistical difference between the two hospitals ( $p=0.007$ ) which was only given at UKS. A statistically significant difference existed with regard to monotherapy between the two hospitals (HVH: 14 patients monotherapy, 8 patients polytherapy; UKS: 4 patients monotherapy, 10 patients polytherapy; $p=0.04$ ). One patient received everolimus due to TSC-related epilepsy in a clinical trial. ${ }^{19}$

\section{Brain Imaging Findings, Treatment, and Response}

In $47 / 54$ (87\%) patients, brain imaging abnormalities were found (-Fig. 2). SEGA was diagnosed in 18/54 (33\%) patients. Everolimus was used in 10 patients with SEGA (6 UKS, 4 $\mathrm{HVH}$ ), and at UKS, 2 patients underwent endoscopic SEGA resection (at 6 and 12 years of age). One patient developed blindness due to severe bilateral papilledema secondary to SEGA growth. The SEGA response was as follows: reduction in SEGA size in 5 patients, unchanged SEGA in four patients, and in SEGA growth was noted in 1 patient.

\section{Neurodevelopmental Findings}

In 28/54 (52\%) patients, a diagnosis of developmental delay was made. Developmental delay was statistically associated with a diagnosis of SEGA and epilepsy ( $p=0.001$ and 0.043 , respectively). A formal intelligence test was performed in five patients (range: 32-103). Neuropsychiatric disorders were diagnosed in $8 / 54(15 \%)$ patients (5 with autism). No significant differences were seen with regard to age between patients with and without neuropsychiatric pathologies.

-Fig. 5 and -Supplementary Table S1 summarize the examinations performed in our cohort. In summary, the following examinations were insufficiently performed and/ or documented: neuropsychiatric evaluation in $41 / 54$ (76\%) patients, renal imaging studies (ultrasound/MRI) in 11/54 (20\%) patients, ophthalmological examination in $15 / 54$ patients (28\%), and dermatological examination in $11 / 54$ (20\%) patients. Similar results were found between the study periods ( - Supplementary Table S2) as well as between both hospitals, except for inadequate dermatological assessment, which was only seen at UKS $(p=0.006)$. 


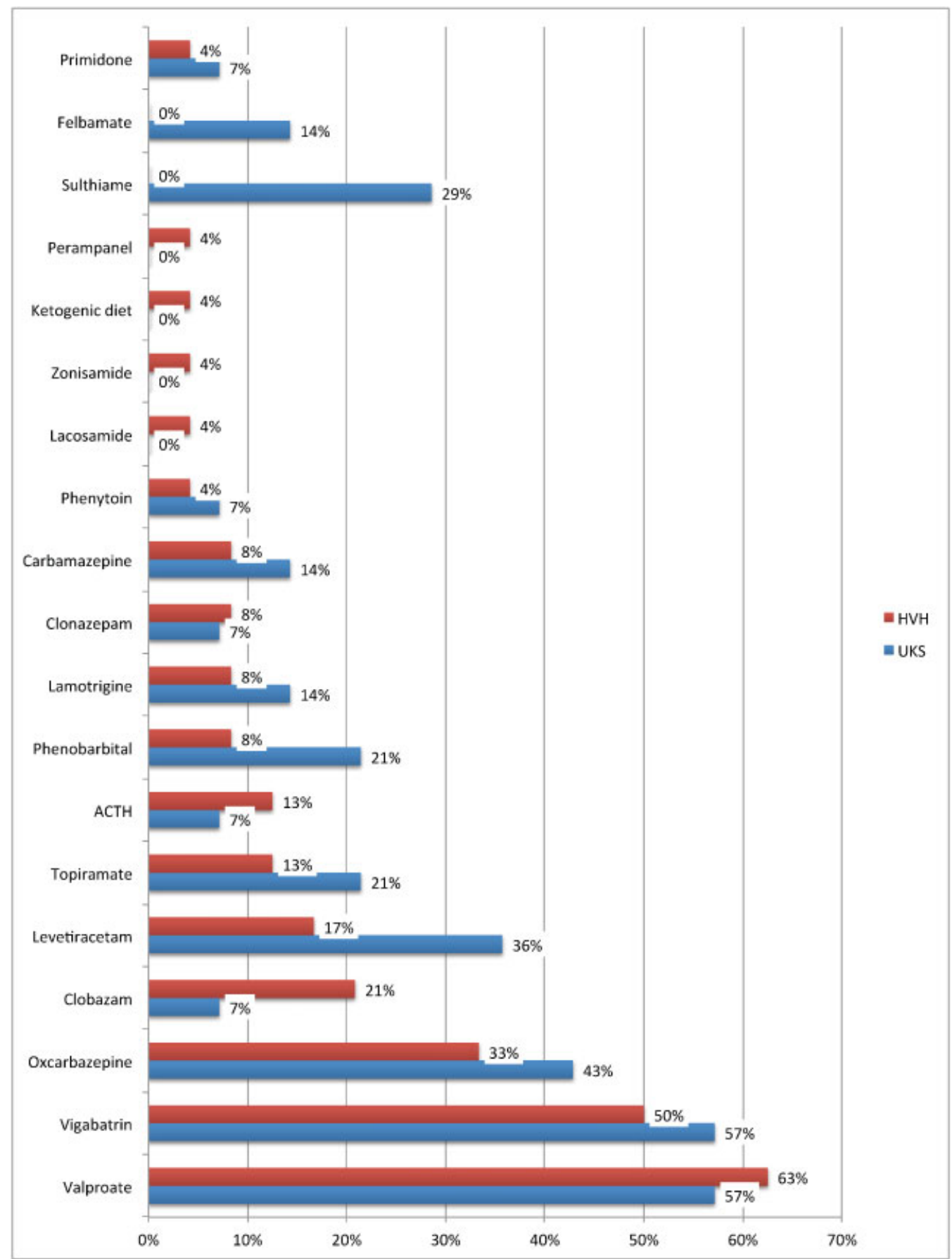

Fig. 3 Summary of use of antiepileptic drugs and ketogenic diet depending on hospital location. ACTH, adrenocorticotropic hormone; HVH, Vall d’Hebron University Hospital; UKS, Saarland University Medical Center.

\section{Discussion}

In our study, all patients fulfilled the current diagnostic criteria for TSC or possible TSC. Due to the retrospective nature of our study, it was only possible to assess whether TSC1 or TSC2 mutations existed with regard to their pathogenicity, thus contributing to establishing a diagnosis of TSC in all affected patients. Of note, in all but two patients, a diagnosis of TSC could be established using clinical criteria only.
No differences were found in terms of patients' demographics between both hospitals. To assess if patients underwent an appropriate diagnostic work-up of the TSC-related manifestations, the published recommendations from 1999 as well as from 2013 were used. ${ }^{6,8}$ Contrary to previous reports, the first key symptom that led to the diagnosis of TSC in our cohort was, for both hospitals, the detection of cardiac rhabdomyoma, most often in utero, followed by epilepsy. ${ }^{20,21}$ Compared with previous reports, a higher rate of in utero detection of cardiac rhabdomyoma was seen in 


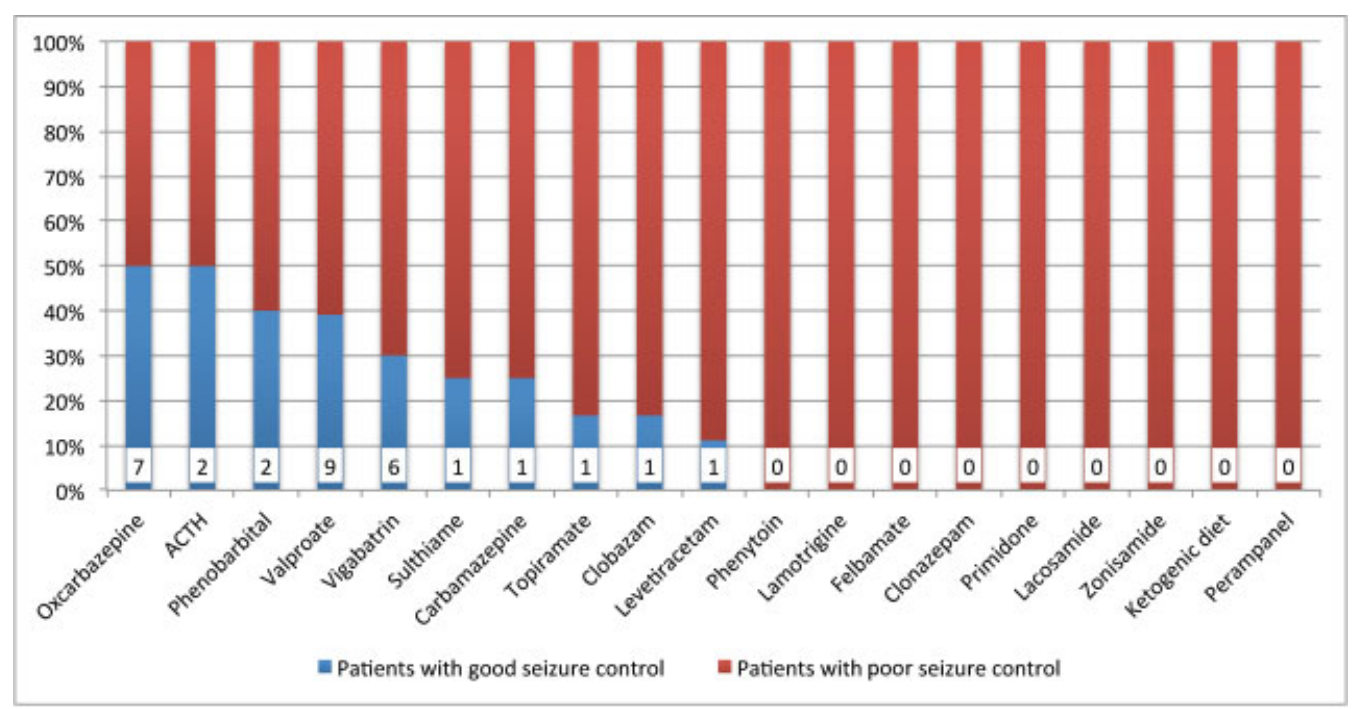

Fig. 4 Summary of antiepileptic drug medication in patients with adequate seizure control. ACTH, adrenocorticotropic hormone.

our two cohorts, indicating improved antenatal care. ${ }^{22}$ We found a lower percentage of patients with epileptic spasms (26\%) compared with other clinical studies (33-54\%) as well as higher percentage of patients with refractory epilepsy (54\%) compared with previous reports (34-44\%). ${ }^{23-27}$ This may be because of the use of different definitions of refractory epilepsy. ${ }^{18}$ According to other studies, valproate and vigabatrin are the most common AEDs used in TSC. ${ }^{28}$ Differences in the use of sulthiame between our two cohorts can be easily explained by a lack of availability of sulthiame in Spain. $^{29}$

In this study, we found a higher prevalence of SEGA compared with other series. ${ }^{17,23,25,30-33}$ This may also be because of differences in SEGA definitions and improvement of cerebral imaging studies, most importantly the widespread use of high-resolution MRI. We found a similar response rate of SEGA to everolimus as in previous reports. ${ }^{34}$ Of note, two patients underwent a neurosurgery procedure,

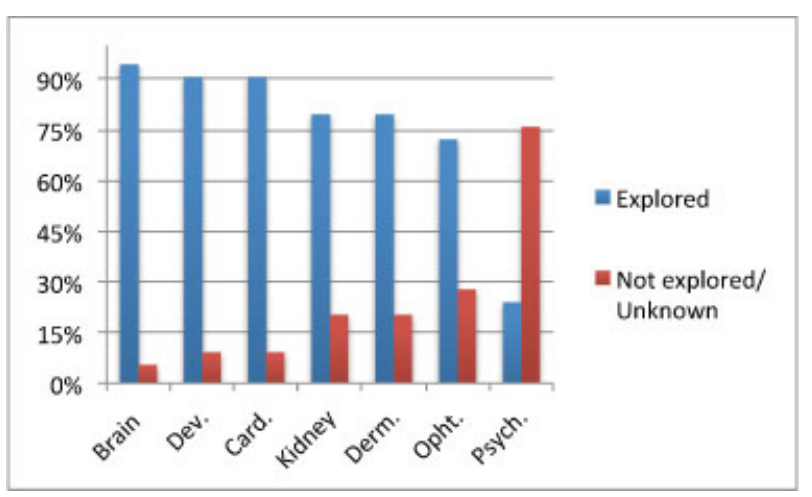

Fig. 5 Examinations performed in the study cohort. Brain, brain imaging; Card., cardiological examination; Derm., dermatological examination; Dev., neurodevelopmental evaluation; Kidney, renal ultrasound; Opht., ophthalmological examination; Psych., neuropsychiatric evaluation. both treated at UKS. This may have to do with differential experiences in the surgical treatment of TSC patients with SEGA. ${ }^{31,35}$ The percentage of patients with developmental delay was similar when compared with previous reports. ${ }^{23,25,33}$ As described previously, a positive correlation between epilepsy and neurodevelopmental delay could be established; however, and contrary to other reports, we also found an association between neurodevelopmental delay and SEGA. ${ }^{26,36}$ Possibly, this association may be indicative of the extent of further underlying CNS pathology, for example, the number of cortical tubers. Unfortunately, due to the retrospective nature of our study, it was not possible to quantify in full detail all CNS pathologies, including the number, size, and location of cortical tubers in our study cohorts.

Of note, this is the first multicenter and international comparison (Spain, Germany) that demonstrates that TSC patients were often not managed in accordance with the current guidelines for diagnosis and long-term treatment. This effect was most apparent with regard to inappropriate assessment of neuropsychiatric, renal, ophthalmological and dermatological manifestations. This pattern was found in both hospitals (HVH and UKS), except for inadequate skin examination, which was only seen at UKS. Of importance, a specific screening test for TSC-associated neuropsychiatric disorders has been published after data completion, thus potentially improving this shortcoming. ${ }^{37}$ We found a higher prevalence of renal AML compared with previous reports. This finding could be explained by neither population demographics nor different prevalence of TSC2 mutations, ${ }^{21,38}$ but may at least in part be also attributed to the use of more sophisticated and readily available imaging modalities (ultrasound). Conversely, a possible explanation for the inappropriate use and implementation of renal examinations may be the young age of our two cohorts: in clinical practice, renal ultrasound is first performed at school age since the occurrence of clinically relevant renal 
angiomyolipoma is usually seen at a later age (school age and thereafter). ${ }^{21,38}$ The percentage of patients with unknown information about dermatological manifestation may also be age-related. However, and of note, hypomelanotic macules (white spots) along with cardiac rhabdomyoma are among the very early organ manifestation of TSC.,39 The underuse of dermatological and ophthalmological examinations may be secondary to the fact that a diagnosis of TSC was often already established based on cerebral imaging or cardiac ultrasound. Therefore, a dermatological and ophthalmological diagnostic evaluation may have been deferred, although recent guidelines recommend repetitive skin assessment. ${ }^{8} \mathrm{At}$ UKS, we have taken action with regard to the aforementioned shortcomings in treating TSC patients, providing comprehensive optimized care in patients with rare diseases at the Center for Rare Diseases (ZSEUKS) at UKS. ${ }^{40}$

Undoubtedly, this study has several weaknesses. First, this is a retrospective analysis with all its inherent shortcomings. Second, given the rarity of this disease, the number of patients included is limited. Third, the inclusion period spans almost four decades with substantial changes in both diagnostic and therapeutic approaches over time. Moreover, as a reference for adequate patient management of TSC patients, we used the definition of Roach et al from 1999, and current surveillance and management recommendations (Krueger and Northrup) were only used in patients included after 2013. ${ }^{6,8}$ Fourth, the cutoff value for inadequate management of TSC patients was somewhat arbitrarily chosen at $>15 \%$.

In summary, this is the first multicenter and international study (Spain, Germany), which demonstrates that TSC patients were often not managed in accordance with current guidelines on diagnosis and long-term treatment. This effect was most relevant with regard to inappropriate assessment of neuropsychiatric, renal, ophthalmological and cutaneous manifestations. More widespread application/use of current guidelines on diagnosis and long-term management has the potential to improve the care of TSC patients.

\section{Ethical Approval}

All procedures performed in studies involving human participants were in accordance with the ethical standards of the institutional and/or national research committee and with the 1964 Helsinki declaration and its later amendments or comparable ethical standards.

\section{Informed Consent}

Informed consent was obtained from all individual participants included in the study.

\section{Conflict of Interest}

Prof. Dr. Macaya-Ruiz reports other from Advisory board, outside the submitted work. All the other authors have no conflict of interest.

\section{Acknowledgments}

The authors received no financial support for this study.

\section{References}

1 Bourneville DM. Sclérose tubéreuse des circonvolutions cérébrales: Idiote et épilepsie hémiplégique. Arch Neurol 1880; 1:81-91

2 Shepherd CW, Gomez MR, Lie JT, Crowson CS. Causes of death in patients with tuberous sclerosis. Mayo Clin Proc 1991;66(08): 792-796

3 Ebrahimi-Fakhari D, Müller CSL, Altmeyer K, et al. Tuberöse Sklerose im Kindes- und Jugendalter. Monatsschr Kinderheilkd 2018;166:65-78. doi:10.1007/s00112-017-0353-6

4 Roach ES, Smith M, Huttenlocher P, Bhat M, Alcorn D, Hawley L. Diagnostic criteria: tuberous sclerosis complex. Report of the Diagnostic Criteria Committee of the National Tuberous Sclerosis Association. J Child Neurol 1992;7(02):221-224

5 Roach ES, Gomez MR, Northrup H. Tuberous sclerosis complex consensus conference: revised clinical diagnostic criteria. J Child Neurol 1998;13(12):624-628

6 Roach ES, DiMario FJ, Kandt RS, Northrup H; National Tuberous Sclerosis Association. Tuberous Sclerosis Consensus Conference: recommendations for diagnostic evaluation. J Child Neurol 1999; 14(06):401-407

7 Northrup H, Krueger DA; International Tuberous Sclerosis Complex Consensus Group. Tuberous sclerosis complex diagnostic criteria update: recommendations of the 2012 International Tuberous Sclerosis Complex Consensus Conference. Pediatr Neurol 2013;49(04):243-254

8 Krueger DA, Northrup H; International Tuberous Sclerosis Complex Consensus Group. Tuberous sclerosis complex surveillance and management: recommendations of the 2012 International Tuberous Sclerosis Complex Consensus Conference. Pediatr Neurol 2013;49(04):255-265

9 European Chromosome 16 Tuberous Sclerosis Consortium. Identification and characterization of the tuberous sclerosis gene on chromosome 16. Cell 1993;75(07):1305-1315

10 van Slegtenhorst M, de Hoogt R, Hermans C, et al. Identification of the tuberous sclerosis gene TSC1 on chromosome 9q34. Science 1997;277(5327):805-808

11 Lebwohl D, Anak O, Sahmoud T, et al. Development of everolimus, a novel oral mTOR inhibitor, across a spectrum of diseases. Ann N Y Acad Sci 2013;1291:14-32

12 Franz DN, Belousova E, Sparagana S, et al. Efficacy and safety of everolimus for subependymal giant cell astrocytomas associated with tuberous sclerosis complex (EXIST-1): a multicentre, randomised, placebo-controlled phase 3 trial. Lancet 2013;381 (9861):125-132

13 Bissler JJ, Kingswood JC, Radzikowska E, et al. Everolimus for angiomyolipoma associated with tuberous sclerosis complex or sporadic lymphangioleiomyomatosis (EXIST-2): a multicentre, randomised, double-blind, placebo-controlled trial. Lancet 2013;381(9869):817-824

14 French JA, Lawson JA, Yapici Z, et al. Adjunctive everolimus therapy for treatment-resistant focal-onset seizures associated with tuberous sclerosis (EXIST-3): a phase 3, randomised, double-blind, placebo-controlled study. Lancet 2016;388(10056):2153-2163

15 Franz DN, Capal JK. mTOR inhibitors in the pharmacologic management of tuberous sclerosis complex and their potential role in other rare neurodevelopmental disorders. Orphanet J Rare Dis 2017;12(01):51

16 Ebrahimi-Fakhari D, Müller CSL, Meyer S, Flotats-Bastardas M, Vogt T, Pföhler C. Topical rapamycin for facial angiofibromas in a child with tuberous sclerosis complex (TSC): a case report and long-term follow-up. Dermatol Ther (Heidelb) 2017;7(01): $175-179$

17 Roth J, Roach ES, Bartels U, et al. Subependymal giant cell astrocytoma: diagnosis, screening, and treatment. Recommendations from the International Tuberous Sclerosis Complex Consensus Conference 2012. Pediatr Neurol 2013;49(06):439-444 
18 Kwan P, Arzimanoglou A, Berg AT, et al. Definition of drug resistant epilepsy: consensus proposal by the ad hoc Task Force of the ILAE Commission on Therapeutic Strategies. Epilepsia 2010;51(06): 1069-1077

19 Krueger DA, Wilfong AA, Holland-Bouley K, et al. Everolimus treatment of refractory epilepsy in tuberous sclerosis complex. Ann Neurol 2013;74(05):679-687

20 Rosser T, Panigrahy A, McClintock W. The diverse clinical manifestations of tuberous sclerosis complex: a review. Semin Pediatr Neurol 2006;13(01):27-36

21 Staley BA, Vail EA, Thiele EA. Tuberous sclerosis complex: diagnostic challenges, presenting symptoms, and commonly missed signs. Pediatrics 2011;127(01):e117-e125

22 Kocabaş A, Ekici F, Cetin II, et al. Cardiac rhabdomyomas associated with tuberous sclerosis complex in 11 children: presentation to outcome. Pediatr Hematol Oncol 2013;30(02):71-79

23 Mettin RR, Merkenschlager A, Bernhard MK, et al. Wide spectrum of clinical manifestations in children with tuberous sclerosis complex-follow-up of 20 children. Brain Dev 2014;36(04):306-314

24 Monteiro T, Garrido C, Pina S, et al. Tuberous sclerosis: clinical characteristics and their relationship to genotype/phenotype [In Spanish]. An Pediatr (Barc) 2014;81(05):289-296

25 Au KS, Williams AT, Roach ES, et al. Genotype/phenotype correlation in 325 individuals referred for a diagnosis of tuberous sclerosis complex in the United States. Genet Med 2007;9(02):88-100

26 Chu-Shore CJ, Major P, Camposano S, Muzykewicz D, Thiele EA. The natural history of epilepsy in tuberous sclerosis complex. Epilepsia 2010;51(07):1236-1241

27 Vignoli A, La Briola F, Turner K, et al. Epilepsy in TSC: certain etiology does not mean certain prognosis. Epilepsia 2013;54(12): 2134-2142

28 Overwater IE, Bindels-de Heus K, Rietman AB, et al. Epilepsy in children with tuberous sclerosis complex: Chance of remission and response to antiepileptic drugs. Epilepsia 2015;56(08):1239-1245

29 Pediamécum. Sultiamo. Comité de Medicamentos de la Asociación Española de Pediatría. http://pediamecum.es/sultiamo. Accessed March 12, 2018

30 Rovira À, Ruiz-Falcó ML, García-Esparza E, et al. Recommendations for the radiological diagnosis and follow-up of neuropatho- logical abnormalities associated with tuberous sclerosis complex. J Neurooncol 2014;118(02):205-223

31 Jóźwiak S, Mandera M, Młynarski W. Natural history and current treatment options for subependymal giant cell astrocytoma in tuberous sclerosis complex. Semin Pediatr Neurol 2015;22(04): 274-281

32 Jóźwiak S, Nabbout R, Curatolo P. Participants of the TSC Consensus Meeting for SEGA and Epilepsy Management. Management of subependymal giant cell astrocytoma (SEGA) associated with tuberous sclerosis complex (TSC): Clinical recommendations. Eur J Paediatr Neurol 2013;17(04):348-352

33 Dabora SL, Jozwiak S, Franz DN, et al. Mutational analysis in a cohort of 224 tuberous sclerosis patients indicates increased severity of TSC2, compared with TSC1, disease in multiple organs. Am J Hum Genet 2001;68(01):64-80

34 Franz DN, Belousova E, Sparagana S, et al. Everolimus for subependymal giant cell astrocytoma in patients with tuberous sclerosis complex: 2-year open-label extension of the randomised EXIST-1 study. Lancet Oncol 2014;15(13):1513-1520

35 Oertel JMK, Baldauf J, Schroeder HWS, Gaab MR. Endoscopic options in children: experience with 134 procedures. J Neurosurg Pediatr 2009;3(02):81-89

36 Kothare SV, Singh K, Hochman T, et al. Genotype/phenotype in tuberous sclerosis complex: associations with clinical and radiologic manifestations. Epilepsia 2014;55(07):1020-1024

37 de Vries PJ, Whittemore VH, Leclezio L, et al. Tuberous sclerosis associated neuropsychiatric disorders (TAND) and the TAND Checklist. Pediatr Neurol 2015;52(01):25-35

38 Kingswood C, Bolton P, Crawford P, et al. The clinical profile of tuberous sclerosis complex (TSC) in the United Kingdom: a retrospective cohort study in the Clinical Practice Research Datalink (CPRD). Eur J Paediatr Neurol 2016;20(02):296-308

39 Ebrahimi-Fakhari D, Meyer S, Vogt T, Pföhler C, Müller CSL. Dermatological manifestations of tuberous sclerosis complex (TSC). J Dtsch Dermatol Ges 2017;15(07):695-700

40 Ebrahimi-Fakhari D, Poryo M, Graf N, et al. Optimized care in patients with rare diseases: TSC at the Center for Rare Diseases (ZSEUKS) at Saarland University Medical Center, Germany. Klin Padiatr 2017;229(06):311-315 